\title{
Blow-up for a parabolic system with nonlocal sources and nonlocal boundary conditions
}

Guangsheng Zhong ${ }^{1,2 *}$ and Lixin Tian ${ }^{2,3}$

\section{*Correspondence:} zhgs917@163.com

'Department of Mathematics, Nantong University, Nantong, 226007, China

${ }^{2}$ Nonlinear Scientific Research Center, Faculty of Science, Jiangsu University, Zhenjiang, 212013, China Full list of author information is available at the end of the article

\begin{abstract}
This paper deals with blow-up properties of solutions to a nonlocal parabolic system with nonlocal boundary conditions. The global existence and finite time blow-up criteria are obtained. Moreover, for some special cases, we establish the precise blow-up rate estimates.
\end{abstract}

Keywords: global existence; finite time blow-up; nonlocal sources; nonlocal boundary conditions; blow-up rate

\section{Introduction}

In this article, we consider the positive solution of the following parabolic equations with nonlocal boundary conditions:

$$
\left\{\begin{array}{l}
u_{t}=f(u)\left(\Delta u+a \int_{\Omega} v d x\right), \quad x \in \Omega, t>0, \\
v_{t}=g(v)\left(\Delta v+b \int_{\Omega} u d x\right), \quad x \in \Omega, t>0, \\
u(x, t)=\int_{\Omega} \phi(x, y) u(y, t) d y, \quad x \in \partial \Omega, t>0, \\
v(x, t)=\int_{\Omega} \psi(x, y) v(y, t) d y, \quad x \in \partial \Omega, t>0, \\
u(x, 0)=u_{0}(x), \quad v(x, 0)=v_{0}(x), \quad x \in \Omega,
\end{array}\right.
$$

where $\Omega$ is a bounded domain in $R^{N}(N \geq 1)$ with smooth boundary $\partial \Omega, a, b>0$, while $\phi(x, y), \psi(x, y)$ are nonnegative and continuous on $\partial \Omega \times \bar{\Omega}, u_{0}(x), v_{0}(x) \in C^{2, \theta}(\bar{\Omega})$ with $0<\theta<1, u_{0}(x), v_{0}(x) \geq 0, u_{0}(x) \not \equiv 0, v_{0}(x) \not \equiv 0$, and satisfy the compatibility conditions

$$
u_{0}(x)=\int_{\Omega} \phi(x, y) u_{0}(y) d y, \quad v_{0}(x)=\int_{\Omega} \psi(x, y) v_{0}(y) d y, \quad x \in \partial \Omega
$$

respectively.

There have been many articles dealing with properties of solutions to degenerate parabolic equations with homogeneous Dirichlet boundary condition (see [1-4] and references therein). For example, Deng et al. [5] studied the parabolic equation with nonlocal source

$$
u_{t}=f(u)\left(\Delta u+a \int_{\Omega} u d x\right), \quad x \in \Omega, t>0,
$$

\section{空 Springer}

( 2015 Zhong and Tian; licensee Springer. This is an Open Access article distributed under the terms of the Creative Commons Attribution License (http://creativecommons.org/licenses/by/4.0), which permits unrestricted use, distribution, and reproduction in any medium, provided the original work is properly credited. 
which is subjected to homogeneous Dirichlet boundary condition. They proved that there exists no global positive solution if $\int^{\infty} 1 /(s f(s)) d s<\infty$ and $\int_{\Omega} \varphi(x) d x>1 / a$, where $\varphi$ is the unique positive solution of the linear elliptic problem

$$
-\Delta \varphi=1, \quad x \in \Omega ; \quad \varphi(x)=0, \quad x \in \partial \Omega .
$$

In [6], Chen and Wang extended the problem (1.2) to the following system:

$$
u_{t}=f(u)\left(\Delta u+a \int_{\Omega} v d x\right), \quad v_{t}=g(v)\left(\Delta v+b \int_{\Omega} u d x\right), \quad x \in \Omega, t>0
$$

with homogeneous Dirichlet boundary condition. Under some conditions, they proved the solution of (1.3) blows up in finite time and even blows up globally.

However, parabolic equations with both nonlocal source and nonlocal boundary condition have been studied as well. For instance, the problem of the following form:

$$
\left\{\begin{array}{l}
u_{t}=f(u)\left(\Delta u+\int_{\Omega} g(u) d x\right), \quad x \in \Omega, t>0, \\
u(x, t)=\int_{\Omega} K(x, y) u^{l}(y, t) d y, \quad x \in \partial \Omega, t>0 \\
u(x, 0)=u_{0}(x), \quad x \in \Omega,
\end{array}\right.
$$

was considered by Lin and Liu [7] for the case $l=1$ and by Zhong and Tian [8] for the case $g(u)=u$. They established global existence and nonexistence of solutions, and they discussed the blow-up properties of solutions.

Porous medium equations with local sources or with nonlocal sources subjected to nonlocal boundary conditions were also studied (see [9-12]). They discussed the conditions of existence and blow-up. For other works on parabolic equations and systems with nonlocal boundary conditions, we refer readers to [13-23] and the references therein.

Motivated by those works above, we will study the problem (1.1) and want to understand how the functions $f(u), g(v)$ and the weight functions $\phi(x, y), \psi(x, y)$ in the boundary condition play substantial roles in determining the blow-up or not of the solutions.

In this article, we make some assumptions on $f(s), g(s)$ as follows:

(H1) $f, g \in C([0, \infty)) \cap C^{1}((0, \infty)), f(0)=g(0)=0$, and $f, g>0, f^{\prime}, g^{\prime} \geq 0$ in $(0, \infty)$.

(H2) Either $\liminf _{s \rightarrow \infty} \frac{f(s)}{g(s)}>0$ or $\liminf _{s \rightarrow \infty} \frac{g(s)}{f(s)}>0$ holds.

In view of the symmetry of the problem, we may suppose that $\liminf _{s \rightarrow \infty} \frac{f(s)}{g(s)}>0$ in $(\mathrm{H} 2)$ throughout this paper. For any $\eta>0$, we can get a constant $K_{0}>0$ such that (see [5])

$$
f(s)>K_{0} g(s) \quad \text { for } s>\eta
$$

Let us introduce the following elliptic problem:

$$
\left\{\begin{array}{l}
-\Delta \varphi_{1}(x)=-\Delta \varphi_{2}(x)=1, \quad x \in \Omega \\
\varphi_{1}(x)=\int_{\Omega} \phi(x, y) \varphi_{1}(y) d y, \quad x \in \partial \Omega \\
\varphi_{2}(x)=\int_{\Omega} \psi(x, y) \varphi_{2}(y) d y, \quad x \in \partial \Omega
\end{array}\right.
$$

where $\int_{\Omega} \phi(x, y) d y<1, \int_{\Omega} \psi(x, y) d y<1$. Then there exists a unique positive solution $\left(\varphi_{1}(x), \varphi_{2}(x)\right)$ of $(1.6)$ (see [19]). 
Define

$$
\mu_{1}=\int_{\Omega} \varphi_{1}(x) d x, \quad \mu_{2}=\int_{\Omega} \varphi_{2}(x) d x .
$$

The main results of this paper are the following theorems.

Theorem 1.1 Suppose that $\int_{\Omega} \phi(x, y) d y<1, \int_{\Omega} \psi(x, y) d y<1$ for any $x \in \partial \Omega$. If $a b \leq \frac{1}{\mu_{1} \mu_{2}}$ or $\int_{s_{0}}^{\infty} \frac{d s}{s f(s)}=\infty$ or $\int_{s_{0}}^{\infty} \frac{d s}{s g(s)}=\infty$ for some $s_{0}>0$, then the solution $(u, v)$ of $(1.1)$ exists globally.

Theorem 1.2 Suppose that $\int_{\Omega} \phi(x, y) d y<1, \int_{\Omega} \psi(x, y) d y<1$ for any $x \in \partial \Omega$. If $a b>\frac{1}{\mu_{1} \mu_{2}}$ and $\int_{s_{0}}^{\infty} \frac{d s}{s g(s)}<\infty$ for some $s_{0}>0$, then the solution $(u, v)$ of $(1.1)$ blows up in finite time.

Theorem 1.3 Suppose that $\int_{\Omega} \phi(x, y) d y \geq 1$ or $\int_{\Omega} \psi(x, y) d y \geq 1$ for any $x \in \partial \Omega$. If $\int_{s_{0}}^{\infty} \frac{d s}{s g(s)}<$ $\infty$ for some $s_{0}>0$, then the solution $(u, v)$ of $(1.1)$ blows up in finite time.

To estimate the blow-up rate, we need an additional assumption on the initial data $u_{0}(x)$, $v_{0}(x)$ :

(H3) There exists a constant $\varepsilon \geq \max \left\{\varepsilon_{1}, \varepsilon_{2}\right\}$, such that

$$
\Delta u_{0}+a \int_{\Omega} v_{0} d x-\varepsilon u_{0}^{k_{1}+1-p} \geq 0, \quad \Delta v_{0}+b \int_{\Omega} u_{0} d x-\varepsilon v_{0}^{k_{2}+1-q} \geq 0,
$$

where $0<p, q<1$ and $\varepsilon_{1}, \varepsilon_{2}, k_{1}, k_{2}$ are given in Section 5 .

Theorem 1.4 Suppose that $\int_{\Omega} \phi(x, y) d y<1, \int_{\Omega} \psi(x, y) d y<1$ for any $x \in \partial \Omega$. Let $f(u)=u^{p}$, $g(v)=v^{q}(0<p, q<1)$. If $(\mathrm{H} 3)$ holds and the solution $(u, v)$ of $(1.1)$ blows up in finite time $T^{*}$. Then there exist positive constants $c_{i}(i=1,2,3,4)$ such that

$$
\begin{aligned}
& c_{1} \leq \max _{x \in \bar{\Omega}} u(x, t)\left(T^{*}-t\right)^{(2-q) /(p+q-p q)} \leq c_{2}, \\
& c_{3} \leq \max _{x \in \bar{\Omega}} v(x, t)\left(T^{*}-t\right)^{(2-p) /(p+q-p q)} \leq c_{4} .
\end{aligned}
$$

This paper is organized as follows. In Section 2, we establish the comparison principle. In Sections 3 and 4, some criteria regarding to global existence and finite time blow-up for (1.1) are given, respectively. In the last section, for some special cases, the blow-up rate estimate is established.

\section{Comparison principle}

We start with the definition of a subsolution and a supersolution of (1.1) and then get to the comparison principle. Set $Q_{T}=\Omega \times(0, T), S_{T}=\partial \Omega \times(0, T)$ and $\bar{Q}_{T}=\bar{\Omega} \times[0, T)$.

Definition 2.1 A vector function $(\underline{u}(x, t), \underline{v}(x, t))$ defined on $\bar{Q}_{T}$, for some $T>0$, is called a subsolution of problem (1.1), if $\underline{u}(x, t), \underline{v}(x, t) \in C\left(\bar{Q}_{T}\right) \cap C^{2,1}\left(Q_{T}\right)$ and satisfy

$$
\left\{\begin{array}{l}
\underline{u}_{t} \leq f(\underline{u})\left(\Delta \underline{u}+a \int_{\Omega} \underline{v} d x\right), \quad x \in \Omega, t>0, \\
\underline{v}_{t} \leq g(\underline{v})\left(\Delta \underline{v}+b \int_{\Omega} \underline{u} d x\right), \quad x \in \Omega, t>0, \\
(\underline{u}, \underline{v}) \leq\left(\int_{\Omega} \phi(x, y) \underline{u}(y, t) d y, \int_{\Omega} \psi(x, y) \underline{v}(y, t) d y\right), \quad x \in \partial \Omega, t>0, \\
(\underline{u}(x, 0), \underline{v}(x, 0)) \leq\left(u_{0}(x), v_{0}(x)\right), \quad x \in \Omega .
\end{array}\right.
$$


Similarly, a vector function $(\bar{u}(x, t), \bar{v}(x, t)) \in\left[C\left(\bar{Q}_{T}\right) \cap C^{2,1}\left(Q_{T}\right)\right]^{2}$ is a supersolution of (1.1) if the reversed inequalities hold in (2.1). A solution of problem (1.1) is a vector function which is both a subsolution and a supersolution of (1.1).

The following comparison principle plays a crucial role in our proofs, which can be obtained by similar arguments to [20,21], and its proof is given here for the sake of completeness.

Lemma 2.1 Suppose that $w(x, t), z(x, t) \in C^{2,1}\left(Q_{T}\right) \cap C\left(\bar{Q}_{T}\right)$ and satisfy

$$
\left\{\begin{array}{l}
w_{t}-d_{1} \Delta w \geq \sum_{j=1}^{n} a_{1 j} w_{x_{j}}+c_{11} w+c_{12} \int_{\Omega} c_{13} z(x, t) d x, \quad(x, t) \in Q_{T}, \\
z_{t}-d_{2} \Delta z \geq \sum_{j=1}^{n} a_{2 j} z_{x_{j}}+c_{21} z+c_{22} \int_{\Omega} c_{23} w(x, t) d x, \quad(x, t) \in Q_{T}, \\
w(x, t) \geq \int_{\Omega} c_{31}(x, y) w(y, t) d y, \quad(x, t) \in S_{T}, \\
z(x, t) \geq \int_{\Omega} c_{32}(x, y) z(y, t) d y, \quad(x, t) \in S_{T}, \\
w(x, 0)>0, \quad z(x, 0)>0, \quad x \in \bar{\Omega},
\end{array}\right.
$$

where $d_{i}=d_{i}(x, t), a_{i j}=a_{i j}(x, t)(i=1,2, j=1,2, \ldots, n)$ and $c_{i j}=c_{i j}(x, t)(i=1,2, j=1,2,3)$ are bounded continuous functions, $d_{i}(x, t), c_{i j}(x, t) \geq 0(i=1,2, j=2,3)$ in $Q_{T}, c_{3 j}(x, y) \geq 0$ $(j=1,2)$ on $\partial \Omega \times \Omega$ and $\int_{\Omega} c_{3 j}(x, y) d y>0$ on $\partial \Omega$. Then $w(x, t), z(x, t)>0$ on $\bar{Q}_{T}$.

Proof Let $\hat{c}_{i}=\sup _{(x, t) \in \bar{Q}_{T}}\left|c_{i 1}\right|, i=1,2$. Set $U=e^{-\gamma t} w, V=e^{-\gamma t} z$ with $\gamma>\max \left\{\hat{c}_{1}, \hat{c}_{2}\right\}$. Then, for $(x, t) \in Q_{T}$, we have

$$
\begin{aligned}
& U_{t}-d_{1} \Delta U \geq \sum_{j=1}^{n} a_{1 j} U_{x_{j}}+\left(c_{11}-\gamma\right) U+c_{12} \int_{\Omega} c_{13} V(x, t) d x, \\
& V_{t}-d_{2} \Delta V \geq \sum_{j=1}^{n} a_{2 j} V_{x_{j}}+\left(c_{21}-\gamma\right) V+c_{22} \int_{\Omega} c_{23} U(x, t) d x .
\end{aligned}
$$

Also

$$
\begin{aligned}
& U \geq \int_{\Omega} c_{31}(x, y) U(y, t) d y, \quad V \geq \int_{\Omega} c_{32}(x, y) V(y, t) d y, \quad(x, t) \in S_{T}, \\
& U(x, 0)=w(x, 0)>0, \quad V(x, 0)=z(x, 0)>0, \quad x \in \bar{\Omega} .
\end{aligned}
$$

It suffices to show that $U, V>0$ on $\bar{Q}_{T}$. Since $U(x, 0), V(x, 0)>0$, there exists $\delta>0$ such that $U, V>0$ for $(x, t) \in \bar{\Omega} \times(0, \delta)$. Suppose for a contradiction that $\bar{t}=\sup \{t \in(0, T)$ : $U, V>0$ on $\bar{\Omega} \times[0, t)\}<T$. Then $U, V \geq 0$ on $\bar{Q}_{\bar{t}}$, and at least one of $U, V$ vanishes at $(\bar{x}, \bar{t})$ for $\bar{x} \in \bar{\Omega}$. Without loss of generality, we assume that $U(\bar{x}, \bar{t})=0=\inf _{(x, t) \in \bar{Q}_{\bar{t}}} U(x, t)$. If $(\bar{x}, \bar{t}) \in Q_{\bar{t}}$, by virtue of the first inequality of (2.3), we get

$$
U_{t}-d_{1} \Delta U \geq \sum_{j=1}^{n} a_{1 j} U_{x_{j}}+\left(c_{11}-\gamma\right) U, \quad(x, t) \in Q_{\bar{t}} .
$$

Then the strong maximum principle implies that $U \equiv 0$ in $Q_{\bar{t}}$, and this is a contradiction. If $(\bar{x}, \bar{t}) \in S_{\bar{t}}$, this results in the contradiction by $(2.4)$ that

$$
0=U(\bar{x}, \bar{t})=e^{-\gamma t} w(\bar{x}, \bar{t})>\int_{\Omega} c_{31}(\bar{x}, y) U(y, \bar{t}) d y>0
$$

due to $\int_{\Omega} c_{31}(x, y) d y>0$ on $\partial \Omega$. This proves $U, V>0$, and in turn $w, z>0$ on $\bar{Q}_{T}$. 
Lemma 2.2 Suppose that $w(x, t), z(x, t) \in C^{2,1}\left(Q_{T}\right) \cap C\left(\bar{Q}_{T}\right)$ and satisfy

$$
\left\{\begin{array}{l}
w_{t}-d_{1} \Delta w \geq \sum_{j=1}^{n} a_{1 j} w_{x_{j}}+c_{11} w+c_{12} \int_{\Omega} c_{13} z(x, t) d x, \quad(x, t) \in Q_{T}, \\
z_{t}-d_{2} \Delta z \geq \sum_{j=1}^{n} a_{2 j} z_{x_{j}}+c_{21} z+c_{22} \int_{\Omega} c_{23} w(x, t) d x, \quad(x, t) \in Q_{T}, \\
w(x, t) \geq \int_{\Omega} c_{31}(x, y) w(y, t) d y, \quad(x, t) \in S_{T}, \\
z(x, t) \geq \int_{\Omega} c_{32}(x, y) z(y, t) d y, \quad(x, t) \in S_{T}, \\
w(x, 0) \geq 0, \quad z(x, 0) \geq 0, \quad x \in \bar{\Omega},
\end{array}\right.
$$

where $d_{i}=d_{i}(x, t), a_{i j}=a_{i j}(x, t)(i=1,2, j=1,2, \ldots, n)$ and $c_{i j}=c_{i j}(x, t)(i=1,2, j=1,2,3)$ are bounded continuous functions, $d_{i}(x, t), c_{i j}(x, t) \geq 0(i=1,2, j=2,3)$ in $Q_{T}, c_{3 j}(x, y) \geq 0$ $(j=1,2)$ on $\partial \Omega \times \Omega$ and $\int_{\Omega} c_{3 j}(x, y) d y>0$ on $\partial \Omega$. Then $w(x, t), z(x, t) \geq 0$ on $\bar{Q}_{T}$.

Proof Set $w(x, t)=\alpha(x) U(x, t), z(x, t)=\alpha(x) V(x, t)$, where $\alpha(x) \in C^{2}(\bar{\Omega})$ satisfies

$\alpha(x)>0 \quad$ on $\bar{\Omega}$;

$$
\alpha(x)=1, \quad \int_{\Omega} \alpha(y) c_{3 j}(x, y) d y \leq \frac{1}{2} \quad \text { on } \partial \Omega, j=1,2 .
$$

A direct computation yields

$$
\begin{gathered}
U_{t}-d_{1} \Delta U \geq \sum_{j=1}^{n}\left(a_{1 j}+d_{1} \frac{2 \alpha_{x_{j}}}{\alpha}\right) U_{x_{j}}+\left(c_{11}+d_{1} \frac{\Delta \alpha}{\alpha}+\sum_{j=1}^{n} a_{1 j} \frac{\alpha_{x_{j}}}{\alpha}\right) U \\
\quad+\frac{c_{12}}{\alpha(x)} \int_{\Omega} c_{13} \alpha(x) V(x, t) d x, \quad(x, t) \in Q_{T}, \\
V_{t}-d_{2} \Delta V \geq \sum_{j=1}^{n}\left(a_{2 j}+d_{2} \frac{2 \alpha_{x_{j}}}{\alpha}\right) V_{x_{j}}+\left(c_{21}+d_{2} \frac{\Delta \alpha}{\alpha}+\sum_{j=1}^{n} a_{2 j} \frac{\alpha_{x_{j}}}{\alpha}\right) V \\
\quad+\frac{c_{22}}{\alpha(x)} \int_{\Omega} c_{23} \alpha(x) U(x, t) d x, \quad(x, t) \in Q_{T}, \\
U \geq \int_{\Omega} c_{31}(x, y) \alpha(y) U(y, t) d y, \quad(x, t) \in S_{T}, \\
V \geq \int_{\Omega} c_{32}(x, y) \alpha(y) V(y, t) d y, \quad(x, t) \in S_{T}, \quad \\
U(x, 0)=w(x, 0) / \alpha(x) \geq 0, \quad V(x, 0)=z(x, 0) / \alpha(x) \geq 0, \quad x \in \bar{\Omega} .
\end{gathered}
$$

Define

$$
\begin{aligned}
& \bar{b}_{i}=\sup _{(x, t) \in Q_{T}}\left|c_{i 1}+d_{i} \frac{\Delta \alpha}{\alpha}+\sum_{j=1}^{n} a_{i j} \frac{\alpha_{x_{j}}}{\alpha}\right|, \\
& \bar{c}_{i}=\sup _{(x, t) \in Q_{T}} \frac{c_{i 2}}{\alpha(x)}, \quad \bar{d}_{i}=\sup _{(x, t) \in Q_{T}} c_{i 3} \alpha(x), \quad i=1,2,
\end{aligned}
$$

and set

$$
\widetilde{U}=U+\tilde{\varepsilon} e^{\gamma t}, \quad \widetilde{V}=V+\tilde{\varepsilon} e^{\gamma t},
$$

with $\gamma>\max \left\{\bar{b}_{1}+\bar{c}_{1} \bar{d}_{1}|\Omega|, \bar{b}_{2}+\bar{c}_{2} \bar{d}_{2}|\Omega|\right\}, \tilde{\varepsilon}>0$. 
Using (2.6), we have

$$
\begin{aligned}
& \widetilde{U}_{t}-d_{1} \Delta \widetilde{U} \geq \sum_{j=1}^{n}\left(a_{1 j}+d_{1} \frac{2 \alpha_{x_{j}}}{\alpha}\right) \widetilde{U}_{x_{j}}+\left(c_{11}+d_{1} \frac{\Delta \alpha}{\alpha}+\sum_{j=1}^{n} a_{1 j} \frac{\alpha_{x_{j}}}{\alpha}\right) \widetilde{U} \\
& \quad+\frac{c_{12}}{\alpha(x)} \int_{\Omega} c_{13} \alpha(x) \widetilde{V}(x, t) d x, \quad(x, t) \in Q_{T}, \\
& \widetilde{V}_{t}-d_{2} \Delta \widetilde{V} \geq \sum_{j=1}^{n}\left(a_{2 j}+d_{2} \frac{2 \alpha_{x_{j}}}{\alpha}\right) \widetilde{V}_{x_{j}}+\left(c_{21}+d_{2} \frac{\Delta \alpha}{\alpha}+\sum_{j=1}^{n} a_{2 j} \frac{\alpha_{x_{j}}}{\alpha}\right) \tilde{V} \\
& \quad+\frac{c_{22}}{\alpha(x)} \int_{\Omega} c_{23} \alpha(x) \widetilde{U}(x, t) d x, \quad(x, t) \in Q_{T}, \\
& \widetilde{U} \geq \int_{\Omega} c_{31}(x, y) \alpha(y) \widetilde{U}(y, t) d y, \quad(x, t) \in S_{T}, \\
& \widetilde{V} \geq \int_{\Omega} c_{32}(x, y) \alpha(y) \widetilde{V}(y, t) d y, \quad(x, t) \in S_{T}, \\
& \widetilde{U}(x, 0)=w(x, 0) / \alpha(x)+\tilde{\varepsilon}>0, \quad \tilde{V}(x, 0)=z(x, 0) / \alpha(x)+\tilde{\varepsilon}>0, \quad x \in \bar{\Omega} .
\end{aligned}
$$

By Lemma 2.1 , we know that $\tilde{U}, \tilde{V}>0$, i.e. $U+\tilde{\varepsilon} e^{\gamma t}>0, V+\tilde{\varepsilon} e^{\gamma t}>0$ on $\bar{Q}_{T}$. It follows by $\tilde{\varepsilon} \rightarrow 0^{+}$that $U, V \geq 0$ and hence $w, z \geq 0$ on $\bar{Q}_{T}$.

By the above lemmas, we obtain the following comparison principle of problem (1.1).

Lemma 2.3 Let ( $\underline{u}, \underline{v})$ and $(\bar{u}, \bar{v})$ be a nonnegative subsolution and supersolution of (1.1) on $\bar{Q}_{T}$, respectively. Then $(\bar{u}, \bar{v}) \geq(\underline{u}, \underline{v})$ on $\bar{Q}_{T}$, if $\bar{u}, \bar{v} \geq \eta$ or $\underline{u}, \underline{v} \geq \eta$ for some small positive constant $\eta$ hold.

The existence of positive classical solutions of (1.1) local in time can be obtained by using the fixed point theorem in [22], and the representation formula and the contraction mapping principle as in [7]. By the above comparison principle, we get the uniqueness of the solution to the problem. The proof is more or less standard, so is omitted here.

\section{Global existence}

In this section, we will give some sufficient conditions for the existence of solution and prove Theorem 1.1.

Proof of Theorem 1.1 Case 1: we assume that $a b \leq \frac{1}{\mu_{1} \mu_{2}}$ holds. Since the functions $\varphi_{1}(x)$, $\varphi_{2}(x)$ are positive and continuous, we can find two large positives constants $k_{1}$ and $k_{2}$ such that

$$
\begin{aligned}
& u_{0}(x) \leq k_{1} \varphi_{1}(x), \quad v_{0}(x) \leq k_{2} \varphi_{2}(x), \quad x \in \bar{\Omega}, \\
& a \mu_{2} \leq k_{1} / k_{2} \leq 1 /\left(b \mu_{1}\right) .
\end{aligned}
$$

Set

$$
w(x, t)=k_{1} \varphi_{1}(x), \quad z(x, t)=k_{2} \varphi_{2}(x) .
$$


Applying (1.6) and (3.1), we get

$$
\begin{aligned}
& w_{t}-f(w)\left(\Delta w+a \int_{\Omega} z d x\right)=-f\left(k_{1} \varphi_{1}\right)\left(-k_{1}+a k_{2} \mu_{2}\right) \geq 0, \quad(x, t) \in Q_{T}, \\
& z_{t}-g(z)\left(\Delta z+b \int_{\Omega} w d x\right)=-g\left(k_{2} \varphi_{2}\right)\left(-k_{2}+b k_{1} \mu_{1}\right) \geq 0, \quad(x, t) \in Q_{T}, \\
& w(x, t)=k_{1} \varphi_{1}(x)=\int_{\Omega} \phi(x, y) w(y, t) d y, \quad(x, t) \in S_{T}, \\
& z(x, t)=\int_{\Omega} \psi(x, y) z(y, t) d y, \quad(x, t) \in S_{T}, \\
& w(x, 0) \geq u_{0}(x), \quad z(x, 0) \geq v_{0}(x), \quad x \in \bar{\Omega} .
\end{aligned}
$$

The above inequalities show that $(w, z)$ is a supersolution of (1.1), and Lemma 2.3 asserts that $(w, z) \geq(u, v)$ for $(x, t) \in \bar{Q}_{T}$. Therefore, the solution $(u, v)$ of (1.1) exists globally.

Case 2: we assume that $\int_{s_{0}}^{\infty} \frac{d s}{s f(s)}=\infty$ holds. It follows from (1.5) that there exists $K_{0}>0$ such that

$$
f(s) \geq K_{0} g(s) \quad \text { for } s>M=\max \left\{\max _{x \in \bar{\Omega}} u_{0}(x), \max _{x \in \bar{\Omega}} v_{0}(x)\right\} .
$$

Choose $A=\max \left\{a|\Omega|, b|\Omega| / K_{0}\right\}$, and consider the ordinary differential equation(ODE)

$$
s^{\prime}(t)=A f(s(t)) s(t), \quad t>0 ; \quad s(0)=M
$$

By the hypothesis (H1) and the theory of ODE, there exists a unique solution $s(t)$ to (3.2), and $s(t)$ is increasing. Since $\int_{s_{0}}^{\infty} \frac{d s}{s f(s)}=\infty$ for some $s_{0}>0, s(t)$ exists globally and $s(t) \geq s_{0}$. Let $w(x, t)=z(x, t)=s(t)$, and note that $\int_{\Omega} \phi(x, y) d y<1, \int_{\Omega} \psi(x, y) d y<1$ on $\partial \Omega$. Then we have

$$
\begin{aligned}
& w_{t}-f(w)\left(\Delta w+a \int_{\Omega} z d x\right) \\
& \quad=s^{\prime}(t)-f(s(t)) a|\Omega| s(t)=(A-a|\Omega|) f(s(t)) s(t) \geq 0, \quad(x, t) \in Q_{T}, \\
& z_{t}-g(z)\left(\Delta z+b \int_{\Omega} w d x\right) \\
& \quad=s^{\prime}(t)-g(s(t)) b|\Omega| s(t) \geq\left(A K_{0}-b|\Omega|\right) g(s(t)) s(t) \geq 0, \quad(x, t) \in Q_{T}, \\
& w(x, t)=s(t)>\int_{\Omega} \phi(x, y) w(y, t) d y, \quad(x, t) \in S_{T}, \\
& z(x, t)>\int_{\Omega} \psi(x, y) z(y, t) d y, \quad(x, t) \in S_{T}, \\
& w(x, 0)=s(0) \geq u_{0}(x), \quad z(x, 0)=s(0) \geq v_{0}(x), \quad x \in \bar{\Omega} .
\end{aligned}
$$

The above inequalities show that $(w, z)$ is a supersolution of problem (1.1). By using Lemma 2.3, we see that the solution $(u, v)$ of $(1.1)$ exists globally.

Case 3: we assume that $\int_{s_{0}}^{\infty} \frac{d s}{s g(s)}=\infty$ holds. We choose two positive constants $l_{1}, l_{2}$ such that

$$
\frac{a|\Omega| l_{2}}{l_{1}}<1, \quad r=\max \left\{1 /\left(l_{1} \min _{x \in \bar{\Omega}} \varphi_{1}(x)\right), 1 / l_{2}\right\} .
$$


Consider the following ODE:

$$
s^{\prime}(t)=\frac{b l_{1} \mu_{1}}{l_{2}} g\left(l_{2} s(t)\right) s(t), \quad t>0 ; \quad s(0)=r(M+1) .
$$

Here $M=\max \left\{\max _{x \in \bar{\Omega}} u_{0}(x), \max _{x \in \bar{\Omega}} v_{0}(x)\right\}$. In view of $\int_{s_{0}}^{\infty} \frac{d s}{s g(s)}=\infty$ for some $s_{0}>0$, we know that $s(t)$ exists globally.

Let

$$
w(x, t)=l_{1} \varphi_{1}(x) s(t), \quad z(x, t)=l_{2} s(t) .
$$

Then by (3.3) we have

$$
\begin{aligned}
& w_{t}-f(w)\left(\Delta w+a \int_{\Omega} z d x\right) \\
& \quad=l_{1} \varphi_{1} s^{\prime}(t)-f\left(l_{1} \varphi_{1} s(t)\right)\left(-l_{1}+a l_{2}|\Omega|\right) s(t) \\
& \quad=l_{1} \varphi_{1} \frac{b l_{1} \mu_{1}}{l_{2}} g\left(l_{2} s(t)\right) s(t)+f\left(l_{1} \varphi_{1} s(t)\right) s(t)\left(l_{1}-a l_{2}|\Omega|\right) \geq 0, \quad(x, t) \in Q_{T}, \\
& z_{t}-g(z)\left(\Delta z+b \int_{\Omega} w d x\right)=l_{2} s^{\prime}(t)-g\left(l_{2} s(t)\right) b l_{1} \mu_{1} s(t)=0, \quad(x, t) \in Q_{T}, \\
& w(x, t)=l_{1} s(t) \int_{\Omega} \phi(x, y) \varphi_{1}(y) d y=\int_{\Omega} \phi(x, y) w(y, t) d y, \quad(x, t) \in S_{T}, \\
& z(x, t)>\int_{\Omega} \psi(x, y) z(y, t) d y, \quad(x, t) \in S_{T}, \\
& w(x, 0)=l_{1} \varphi_{1}(x) s(0)>u_{0}(x), \quad z(x, 0)=l_{2} s(0)>v_{0}(x), \quad x \in \bar{\Omega} .
\end{aligned}
$$

These formulas show that $(w, z)$ is a supersolution of $(1.1)$. Therefore, $(w, z) \geq(u, v)$. Since $(w, z)$ exists globally, so does $(u, v)$. This completes the proof.

\section{Blow-up results}

In this section, we assume that $(u, v)$ is a positive solution of $(1.1)$ on $\bar{Q}_{T}$, where $T$ is the maximal existence time.

Proof of Theorem 1.2 Set $\underline{K}_{i}=\min _{x \in \bar{\Omega}} \varphi_{i}(x), \bar{K}_{i}=\max _{x \in \bar{\Omega}} \varphi_{i}(x), i=1$, 2. In view of $a b>\frac{1}{\mu_{1} \mu_{2}}$, then there exist positive constants $l_{1}, l_{2}>1$ such that

$$
l_{1} \underline{K}_{1}, l_{2} \underline{K}_{2} \geq 1 \text { and } \quad \frac{1}{b \mu_{1}}<\frac{l_{1}}{l_{2}}<a \mu_{2} .
$$

Taking $\delta=\frac{1}{2} \min \left\{\min _{x \in \bar{\Omega}} u_{0}(x), \min _{x \in \bar{\Omega}} v_{0}(x)\right\}$ and $r=\min \left\{\frac{1}{l_{1} \bar{K}_{1}}, \frac{1}{l_{2} \bar{K}_{2}}\right\}$, it follows from (1.5) that there exists $K_{0}^{\prime}>0$ such that

$$
f(s) \geq K_{0}^{\prime} g(s) \quad \text { for } s>r \delta .
$$

Choose $B=\min \left\{\frac{K_{0}^{\prime}\left(a l_{2} \mu_{2}-l_{1}\right)}{l_{1} \bar{K}_{1}}, \frac{b l_{1} \mu_{1}-l_{2}}{l_{2} \bar{K}_{2}}\right\}$, and consider the following ODE:

$$
s^{\prime}(t)=B g(s(t)) s(t), \quad t>0 ; \quad s(0)=r \delta .
$$

Since $\int_{s_{0}}^{\infty} \frac{d s}{s g(s)}<\infty$ for some $s_{0}>0, s(t)$ blows up in finite time. 
Let

$$
w(x, t)=l_{1} \varphi_{1}(x) s(t), \quad z(x, t)=l_{2} \varphi_{2}(x) s(t) .
$$

Then (4.1) and (4.2) imply that

$$
\begin{array}{rl}
w_{t} & f(w)\left(\Delta w+a \int_{\Omega} z d x\right) \\
& =l_{1} \varphi_{1} s^{\prime}(t)-f\left(l_{1} \varphi_{1} s(t)\right)\left(-l_{1}+a l_{2} \mu_{2}\right) s(t) \\
& \leq l_{1} \bar{K}_{1} B g(s(t)) s(t)-K_{0}^{\prime} g\left(l_{1} \varphi_{1} s(t)\right)\left(-l_{1}+a l_{2} \mu_{2}\right) s(t) \\
& \leq\left(l_{1} \bar{K}_{1} B-K_{0}^{\prime}\left(a l_{2} \mu_{2}-l_{1}\right)\right) g(s(t)) s(t) \leq 0, \quad(x, t) \in Q_{T}, \\
z_{t} & -g(z)\left(\Delta z+b \int_{\Omega} w d x\right) \\
& =l_{2} \varphi_{2} s^{\prime}(t)-g\left(l_{2} \varphi_{2} s(t)\right)\left(-l_{2}+b l_{1} \mu_{1}\right) s(t) \\
& \leq l_{2} \bar{K}_{2} B g(s(t)) s(t)-g\left(l_{2} \varphi_{2} s(t)\right)\left(b l_{1} \mu_{1}-l_{2}\right) s(t) \\
& \leq\left(l_{2} \bar{K}_{2} B-\left(b l_{1} \mu_{1}-l_{2}\right)\right) g(s(t)) s(t) \leq 0, \quad(x, t) \in Q_{T}, \\
w & (x, t)=\int_{\Omega} \phi(x, y) w(y, t) d y, \quad z(x, t)=\int_{\Omega} \psi(x, y) z(y, t) d y, \quad(x, t) \in S_{T}, \\
w(x, 0)=l_{1} \varphi_{1}(x) s(0)<u_{0}(x), \quad z(x, 0)=l_{2} \varphi_{2}(x) s(0)<v_{0}(x), \quad x \in \bar{\Omega} .
\end{array}
$$

The above inequalities imply that $(w, z)$ is a subsolution of $(1.1)$, so $(w, z) \leq(u, v)$. Due to $(w, z)$ blowing up in finite time, $(u, v)$ blows up in finite time, and this completes the proof.

Proof of Theorem 1.3 (i) Suppose that $\int_{\Omega} \phi(x, y) d y \geq 1, \int_{\Omega} \psi(x, y) d y \geq 1$ on $\partial \Omega$. By (1.5), there exists a positive constant $K_{0}^{\prime \prime}>0$ such that

$$
f(s) \geq K_{0}^{\prime \prime} g(s) \quad \text { for } s>m=\frac{1}{2} \min \left\{\min _{x \in \bar{\Omega}} u_{0}(x), \min _{x \in \bar{\Omega}} v_{0}(x)\right\} .
$$

Let $C=\min \left\{a K_{0}^{\prime \prime}|\Omega|, b|\Omega|\right\}$, and consider the following ODE:

$$
s^{\prime}(t)=C g(s(t)) s(t), \quad t>0 ; \quad s(0)=m .
$$

Since $\int_{s_{0}}^{\infty} \frac{d s}{s g(s)}<\infty$ for some $s_{0}>0$, the solution $s(t)$ of (4.3) blows up.

Let $w(x, t)=z(x, t)=s(t)$, then we obtain

$$
\begin{aligned}
w_{t} & -f(w)\left(\Delta w+a \int_{\Omega} z d x\right) \\
& =s^{\prime}(t)-f(s(t)) a|\Omega| s(t)=C g(s(t)) s(t)-a|\Omega| f(s(t)) s(t) \\
& \leq\left(C-a K_{0}^{\prime \prime}|\Omega|\right) g(s(t)) s(t) \leq 0, \quad(x, t) \in Q_{T}, \\
z_{t} & -g(z)\left(\Delta z+b \int_{\Omega} w d x\right)
\end{aligned}
$$




$$
\begin{aligned}
& =s^{\prime}(t)-g(s(t)) b|\Omega| s(t)=(C-b|\Omega|) g(s(t)) s(t) \leq 0, \quad(x, t) \in Q_{T}, \\
& w(x, t)=s(t) \leq \int_{\Omega} \phi(x, y) w(y, t) d y, \quad(x, t) \in S_{T}, \\
& z(x, t) \leq \int_{\Omega} \psi(x, y) z(y, t) d y, \quad(x, t) \in S_{T}, \\
& w(x, 0)=s(0) \leq u_{0}(x), \quad z(x, 0)=s(0) \leq v_{0}(x), \quad x \in \bar{\Omega} .
\end{aligned}
$$

The above inequalities show that $(w, z)$ is a subsolution of problem (1.1), and Lemma 2.3 shows that $(w, z) \leq(u, v)$, so the solution $(u, v)$ of (1.1) blows up in finite time.

(ii) Suppose that $\int_{\Omega} \phi(x, y) d y<1, \int_{\Omega} \psi(x, y) d y \geq 1$ on $\partial \Omega$. Let $\lambda>0$ be the first eigenvalue of the eigenvalue problem

$$
-\Delta \Phi=\lambda \Phi, \quad x \in \Omega ; \quad \Phi(x)=0, \quad x \in \partial \Omega
$$

and $\Phi(x)$ be the corresponding eigenfunction with $\max _{x \in \bar{\Omega}} \Phi(x)=1, \Phi(x)>0$ in $\Omega$. Set $\underline{K}=\min _{x \in \Omega} \Phi(x)>0, \varepsilon_{*}$ is a small enough positive constant such that $\varepsilon_{*}<\min \left\{\frac{a|\Omega|}{\lambda}, \frac{1}{\underline{K}}\right\}$ and $s(t)$ is the solution of ODE as follows:

$$
s^{\prime}(t)=D g\left(\varepsilon_{*} \underline{K} s(t)\right) s(t), \quad t>0 ; \quad s(0)=\varepsilon_{*} \underline{K} m,
$$

where $D=\min \left\{\frac{a|\Omega|-\varepsilon_{*} \lambda}{\varepsilon_{*} K_{0}^{\prime \prime \prime}}, b \varepsilon_{*} \underline{K}|\Omega|\right\}, m$ is given at the beginning of this proof, and $K_{0}^{\prime \prime \prime}>0$ is determined by (1.5) and satisfies $f(s)>K_{0}^{\prime \prime \prime} g(s)$ for $s \geq \varepsilon_{*} \underline{K} m$. Since $\int_{s_{0}}^{\infty} \frac{d s}{s g(s)}<\infty$ for some $s_{0}>0$, the solution to (4.5) blows up in finite time.

Let

$$
w(x, t)=\varepsilon_{*} \Phi(x) s(t), \quad z(x, t)=s(t) .
$$

By (4.4), we have

$$
\begin{aligned}
& w_{t}-f(w)\left(\Delta w+a \int_{\Omega} z d x\right) \\
&=\varepsilon_{*} \Phi(x) s^{\prime}(t)-f\left(\varepsilon_{*} \Phi(x) s(t)\right)\left(-\varepsilon_{*} \lambda \Phi(x) s(t)+a|\Omega| s(t)\right) \\
&=\varepsilon_{*} \Phi(x) D g\left(\varepsilon_{*} \underline{K} s(t)\right) s(t)-\left(a|\Omega|-\varepsilon_{*} \lambda \Phi(x)\right) f\left(\varepsilon_{*} \Phi(x) s(t)\right) s(t) \\
& \leq\left[\varepsilon_{*} D K_{0}^{\prime \prime \prime}-\left(a|\Omega|-\varepsilon_{*} \lambda\right)\right] f\left(\varepsilon_{*} \Phi(x) s(t)\right) s(t) \leq 0, \quad(x, t) \in Q_{T}, \\
& z_{t}-g(z)\left(\Delta z+b \int_{\Omega} w d x\right) \\
&=s^{\prime}(t)-g(s(t)) b \varepsilon_{*} s(t) \int_{\Omega} \Phi d x \\
& \leq D g\left(\varepsilon_{*} \underline{K} s(t)\right) s(t)-b \varepsilon_{*} \underline{K}|\Omega| g(s(t)) s(t) \\
& \leq\left(D-b \varepsilon_{*} \underline{K}|\Omega|\right) g(s(t)) s(t) \leq 0, \quad(x, t) \in Q_{T}, \\
& w(x, t)=0 \leq \int_{\Omega} \phi(x, y) w(y, t) d y, \quad z(x, t) \leq \int_{\Omega} \psi(x, y) z(y, t) d y, \quad(x, t) \in S_{T}, \\
& w(x, 0)=\varepsilon_{*} \Phi(x) s(0) \leq \varepsilon_{*}^{2} \underline{K} m \leq u_{0}(x), \quad z(x, 0)=s(0) \leq v_{0}(x), \quad x \in \bar{\Omega} .
\end{aligned}
$$


All the above inequalities show that $(w, z)=\left(\varepsilon_{*} \Phi(x) s(t), s(t)\right)$ is a subsolution of (1.1). By Lemma 2.3, we know $(u, v) \geq(w, z)$. Since $(w, z)$ blows up in finite time, so does $(u, v)$.

(iii) Suppose that $\int_{\Omega} \phi(x, y) d y \geq 1, \int_{\Omega} \psi(x, y) d y<1$ on $\partial \Omega$. In this case, the proof can be treated as case (ii), so we omit it here. This completes the proof of Theorem 1.3.

\section{Blow-up rate estimates}

Now, we will consider the blow-up rate of the solution to (1.1) in the special case that $f(u)=u^{p}, g(v)=v^{q}(0<p, q<1)$ and $\int_{\Omega} \phi(x, y) d y<1, \int_{\Omega} \psi(x, y) d y<1$ for any $x \in \partial \Omega$, i.e.

$$
\left\{\begin{array}{l}
u_{t}=u^{p}\left(\Delta u+a \int_{\Omega} v d x\right), \quad x \in \Omega, t>0, \\
v_{t}=v^{q}\left(\Delta v+b \int_{\Omega} u d x\right), \quad x \in \Omega, t>0, \\
u(x, t)=\int_{\Omega} \phi(x, y) u(y, t) d y, \quad x \in \partial \Omega, t>0, \\
v(x, t)=\int_{\Omega} \psi(x, y) v(y, t) d y, \quad x \in \partial \Omega, t>0, \\
u(x, 0)=u_{0}(x), \quad v(x, 0)=v_{0}(x), \quad x \in \Omega .
\end{array}\right.
$$

It can be seen from Theorem 1.2 that the solution $(u, v)$ to (5.1) blows up in finite time $T^{*}$. Denote $U(t)=\max _{x \in \bar{\Omega}} u(x, t), V(t)=\max _{x \in \bar{\Omega}} v(x, t)$, which are Lipschitz continuous (see [23] or [12]). From (5.1), we have $U(t), V(t)$ satisfying

$$
U_{t} \leq a|\Omega| U^{p} V, \quad V_{t} \leq b|\Omega| U V^{q}, \quad \text { a.e. } t \in\left(0, T^{*}\right) .
$$

Let $\rho_{1}=2-p, \rho_{2}=2-q$, by virtue of Young's inequality, there exists $C_{1}>0$ such that

$$
\left(U^{\rho_{1}}+V^{\rho_{2}}\right)_{t} \leq\left(a \rho_{1}+b \rho_{2}\right)|\Omega|\left(U^{\rho_{1}}\right)^{1 / \rho_{1}}\left(V^{\rho_{2}}\right)^{1 / \rho_{2}} \leq C_{1}\left(U^{\rho_{1}}+V^{\rho_{2}}\right)^{\left(\rho_{1}+\rho_{2}\right) / \rho_{1} \rho_{2}} .
$$

Integrating the above inequality over $\left(t, T^{*}\right)$, we can get

$$
U^{\rho_{1}}(t)+V^{\rho_{2}}(t) \geq C_{2}\left(T^{*}-t\right)^{-\rho_{1} \rho_{2} / d},
$$

where $C_{2}>0$ is a constant and $d=1-(1-p)(1-q)$. Let $k_{1}=d / \rho_{2}, k_{2}=d / \rho_{1}$.

Lemma 5.1 There exists a constant $\varepsilon>0$, which is defined in (H3) such that

$$
u_{t}-\varepsilon u^{k_{1}+1} \geq 0, \quad v_{t}-\varepsilon v^{k_{2}+1} \geq 0, \quad(x, t) \in \Omega \times\left(0, T^{*}\right) .
$$

Proof Set $J_{1}(x, t)=u_{t}-\varepsilon u^{k_{1}+1}, J_{2}(x, t)=v_{t}-\varepsilon v^{k_{2}+1}$. A series of computation yields

$$
\begin{aligned}
J_{1 t} & u^{p} \Delta J_{1}-2 p \varepsilon u^{k_{1}} J_{1}-a u^{p} \int_{\Omega} J_{2} d x \\
= & p u^{-1} J_{1}^{2}+\varepsilon\left(k_{1}+1\right) k_{1} u^{k_{1}+p-1}|\nabla u|^{2}+p \varepsilon^{2} u^{2 k_{1}+1} \\
& +a \varepsilon u^{p} \int_{\Omega} v^{k_{2}+1} d x-a \varepsilon\left(k_{1}+1\right) u^{k_{1}+p} \int_{\Omega} v d x \\
\geq & p \varepsilon^{2} u^{2 k_{1}+1}+a \varepsilon u^{p} \int_{\Omega} v^{k_{2}+1} d x-a \varepsilon\left(k_{1}+1\right) u^{k_{1}+p} \int_{\Omega} v d x \\
= & a \varepsilon u^{p}\left[(p \varepsilon / a) u^{2 k_{1}+1-p}+\int_{\Omega} v^{k_{2}+1} d x-\left(k_{1}+1\right) u^{k_{1}} \int_{\Omega} v d x\right] .
\end{aligned}
$$


Since $\frac{k_{1}}{2 k_{1}+1-p}+\frac{1}{k_{2}+1}=1$, then the Hölder inequality and Young's inequality imply

$$
\begin{aligned}
u^{k_{1}} \int_{\Omega} v d x & \leq|\Omega|^{k_{2} /\left(k_{2}+1\right)} u^{k_{1}}\left(\int_{\Omega} v^{k_{2}+1} d x\right)^{1 /\left(k_{2}+1\right)} \\
& \leq|\Omega|^{k_{2} /\left(k_{2}+1\right)}\left[\frac{k_{1}}{2 k_{1}+1-p}\left(\sigma u^{k_{1}}\right)^{\left(2 k_{1}+1-p\right) / k_{1}}+\frac{1}{k_{2}+1} \sigma^{-\left(k_{2}+1\right)} \int_{\Omega} v^{k_{2}+1} d x\right]
\end{aligned}
$$

where $\sigma=\left(\frac{k_{1}+1}{k_{2}+1}\right)^{1 /\left(k_{2}+1\right)}|\Omega|^{k_{2} /\left(k_{2}+1\right)^{2}}$. Taking $\varepsilon_{1}=\frac{a k_{2}}{p}\left(\frac{k_{1}+1}{k_{2}+1}\right)^{1+\left(1 / k_{2}\right)}|\Omega|$, then

$$
J_{1 t}-u^{p} \Delta J_{1}-2 p \varepsilon u^{k_{1}} J_{1}-a u^{p} \int_{\Omega} J_{2} d x \geq p \varepsilon\left(\varepsilon-\varepsilon_{1}\right) u^{2 k_{1}+1} \geq 0
$$

Similarly, we can determine a number $\varepsilon_{2}=\frac{b k_{1}}{q}\left(\frac{k_{2}+1}{k_{1}+1}\right)^{1+\left(1 / k_{1}\right)}|\Omega|$ satisfying

$$
J_{2 t}-v^{q} \Delta J_{2}-2 q \varepsilon v^{k_{2}} J_{2}-b v^{q} \int_{\Omega} J_{1} d x \geq q \varepsilon\left(\varepsilon-\varepsilon_{2}\right) v^{2 k_{2}+1} \geq 0 .
$$

For $(x, t) \in \partial \Omega \times\left(0, T^{*}\right)$, we have

$$
\begin{aligned}
J_{1}(x, t) & =u_{t}-\varepsilon u^{k_{1}+1} \\
& =\int_{\Omega} \phi(x, y) u_{t}(y, t) d y-\varepsilon\left(\int_{\Omega} \phi(x, y) u(y, t) d y\right)^{k_{1}+1} \\
& =\int_{\Omega} \phi(x, y) J_{1}(y, t) d y+\varepsilon\left[\int_{\Omega} \phi(x, y) u^{k_{1}+1}(y, t) d y-\left(\int_{\Omega} \phi(x, y) u(y, t) d y\right)^{k_{1}+1}\right] .
\end{aligned}
$$

Using the Hölder inequality and noting that $\int_{\Omega} \phi(x, y) d y<1$, we have

$$
\begin{aligned}
& \int_{\Omega} \phi(x, y) u^{k_{1}+1}(y, t) d y-\left(\int_{\Omega} \phi(x, y) u(y, t) d y\right)^{k_{1}+1} \\
& \quad \geq \int_{\Omega} \phi(x, y) u^{k_{1}+1}(y, t) d y\left[1-\left(\int_{\Omega} \phi(x, y) d y\right)^{k_{1}}\right] \geq 0 .
\end{aligned}
$$

Hence

$$
J_{1}(x, t) \geq \int_{\Omega} \phi(x, y) J_{1}(y, t) d y .
$$

By a similar argument, we have

$$
J_{2}(x, t) \geq \int_{\Omega} \psi(x, y) J_{2}(y, t) d y
$$

On the other hand, (H3) implies that $J_{1}(x, 0) \geq 0, J_{2}(x, 0) \geq 0, x \in \Omega$. By Lemma 2.2, we have $J_{1} \geq 0, J_{2} \geq 0$. This completes the proof.

It follows from (5.4) that

$$
U_{t}-\varepsilon U^{k_{1}+1} \geq 0, \quad V_{t}-\varepsilon V^{k_{2}+1} \geq 0, \quad(x, t) \in \Omega \times\left(0, T^{*}\right) .
$$


Integrating (5.5) from $\left(t, T^{*}\right)$, we conclude that

$$
U(t) \leq\left(\varepsilon k_{1}\right)^{-1 / k_{1}}\left(T^{*}-t\right)^{-1 / k_{1}}, \quad V(t) \leq\left(\varepsilon k_{2}\right)^{-1 / k_{2}}\left(T^{*}-t\right)^{-1 / k_{2}} .
$$

Combining (5.5) with (5.2), we can obtain

$$
\left(\frac{\varepsilon}{a|\Omega|}\right)^{\rho_{2}} U^{\rho_{1}} \leq V^{\rho_{2}}, \quad\left(\frac{\varepsilon}{b|\Omega|}\right)^{\rho_{1}} V^{\rho_{2}} \leq U^{\rho_{1}} .
$$

From (5.3) and (5.7), we conclude that

$$
\begin{aligned}
& U(t) \geq C_{2}^{1 / \rho_{1}}\left[1+\left(\frac{\varepsilon}{b|\Omega|}\right)^{-\rho_{1}}\right]^{-1 / \rho_{1}}\left(T^{*}-t\right)^{-1 / k_{1}}, \\
& V(t) \geq C_{2}^{1 / \rho_{2}}\left[1+\left(\frac{\varepsilon}{a|\Omega|}\right)^{-\rho_{2}}\right]^{-1 / \rho_{2}}\left(T^{*}-t\right)^{-1 / k_{2}} .
\end{aligned}
$$

By (5.6) and (5.8), we can obtain Theorem 1.4 immediately.

\section{Competing interests}

The authors declare that they have no competing interests.

\section{Authors' contributions}

All the authors contributed equally and significantly in writing this article. All the authors read and approved the final manuscript.

\section{Author details}

'Department of Mathematics, Nantong University, Nantong, 226007, China. ${ }^{2}$ Nonlinear Scientific Research Center, Faculty of Science, Jiangsu University, Zhenjiang, 212013, China. ${ }^{3}$ Nanjing Normal University, Nanjing, 210097, China.

\section{Acknowledgements}

The authors would like to express their sincere gratitude to the anonymous reviewers for their helpful comments and suggestions on the manuscript. This work is supported by the National Natural Science Foundation of China (Nos. 11171135, 51276081), and the Major Project of Natural Science Foundation of Jiangsu Province Colleges and Universities (14KJA110001).

\section{Received: 25 September 2014 Accepted: 6 April 2015 Published online: 16 April 2015}

\section{References}

1. Souplet, P: Uniform blow-up profiles and boundary for diffusion equations with nonlocal nonlinear source. J. Differ. Equ. 153, 374-406 (1999)

2. Weissler, FB: An $L^{\infty}$ blow-up estimate for a nonlinear heat equation. Commun. Pure Appl. Math. 38, $291-296$ (1985)

3. Galaktionov, VA: On asymptotic self-similar behavior for a quasilinear heat equation: single point blow-up. SIAM J. Math. Anal. 26, 675-693 (1995)

4. Giga, Y, Umeda, N: Blow-up directions at space infinity for solutions of semilinear heat equations. Bol. Soc. Parana. Mat. 23, 9-28 (2005)

5. Deng, WB, Li, YX, Xie, CH: Existence and nonexistence of global solutions of some nonlocal degenerate parabolic equations. Appl. Math. Lett. 16, 803-808 (2003)

6. Chen, YJ, Wang, MX: A class of nonlocal and degenerate quasilinear parabolic system not in divergence form. Nonlinear Anal. 71, 3530-3537 (2009)

7. Lin, ZG, Liu, YR: Uniform blowup profiles for diffusion equations with nonlocal source and nonlocal boundary. Acta Math. Sci., Ser. B 24, 443-450 (2004)

8. Zhong, GS, Tian, LX: Blow up problems for a degenerate parabolic equation with nonlocal source and nonlocal nonlinear boundary condition. Bound. Value Probl. 2012, 45 (2012)

9. Wang, YL, Mu, CL, Xiang, ZY: Blowup of solutions to a porous medium equation with nonlocal boundary condition. Appl. Math. Comput. 192, 579-585 (2007)

10. Cui, ZJ, Yang, ZD: Roles of weight functions to a nonlinear porous medium equation with nonlocal source and nonlocal boundary condition. J. Math. Anal. Appl. 342, 559-570 (2008)

11. Han, YZ, Gao, WJ: A porous medium equation with nonlocal boundary condition and a localized source. Appl. Anal. 3, 601-613 (2012)

12. Chen, YP: Global blow-up for a localized quasilinear parabolic system with nonlocal boundary conditions. Appl. Anal. 7, 1495-1510 (2013)

13. Gladkov, A, Kim, Kl: Uniqueness and nonuniqueness for reaction-diffusion equation with nonlinear nonlocal boundary condition. Adv. Math. Sci. Appl. 19, 39-49 (2009) 
14. Gladkov, A, Guedda, M: Blow-up problem for semilinear heat equation with absorption and a nonlocal boundary condition. Nonlinear Anal. 74, 4573-4580 (2011)

15. Liang, J, Wang, HY, Xiao, TJ: On a comparison principle for delay coupled systems with nonlocal and nonlinear boundary conditions. Nonlinear Anal. 71, 359-365 (2009)

16. Liu, DM, Mu, CL: Blowup properties for a semilinear reaction-diffusion system with nonlinear nonlocal boundary conditions. Abstr. Appl. Anal. 2010, Article ID 148035 (2010)

17. $\mathrm{Mu}, \mathrm{CL}, \mathrm{Liu}, \mathrm{DM}$, Zhou, SM: Properties of positive solutions for a nonlocal reaction diffusion equation with nonlocal nonlinear boundary condition. J. Korean Math. Soc. 47, 1317-1328 (2011)

18. Gladkov, A, Nikitin, A: A reaction-diffusion system with nonlinear nonlocal boundary conditions. Int. J. Partial Differ. Equ. 2014, Article ID 523656 (2014)

19. Chen, YP, Liu, LH: Global blow-up for a localized nonlinear parabolic equation with a nonlocal boundary condition. J. Math. Anal. Appl. 384, 421-430 (2011)

20. Zheng, SN, Kong, LH: Roles of weight functions in a nonlinear nonlocal parabolic system. Nonlinear Anal. 68 , 2406-2416 (2008)

21. Wang, MX, Wang, YM: Properties of positive solutions for non-local reaction-diffusion problems. Math. Methods Appl. Sci. 19, 1141-1156 (1996)

22. Yin, HM: On a class of parabolic equations with nonlocal boundary conditions. J. Math. Anal. Appl. 294, 712-728 (2004)

23. Friedman, A, McLeod, B: Blow-up of positive solutions of semilinear heat equations. Indiana Univ. Math. J. 34, 425-447 (1985)

\section{Submit your manuscript to a SpringerOpen ${ }^{\mathcal{O}}$ journal and benefit from:}

- Convenient online submission

- Rigorous peer review

- Immediate publication on acceptance

- Open access: articles freely available online

- High visibility within the field

- Retaining the copyright to your article 